\title{
Role of bariatric surgery in treatment of non-alcoholic fatty liver disease
}

\author{
Mohammad Kermansaravi, ${ }^{a, b}$ Mohammad Ebrahimian, ${ }^{c}$ Delaram Delbari, ${ }^{c}$ Simin Khamoushi, ${ }^{c}$ and Ali Kabira
}

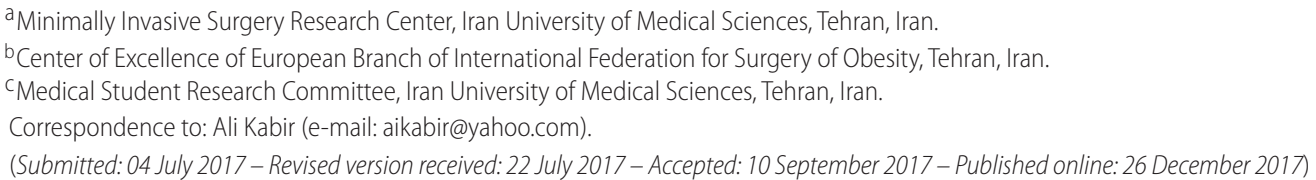

The prevalence of non-alcoholic fatty liver disease (NAFLD) and non-alcoholic steatohepatitis (NASH) is increasing due to increasing of
morbid obesity prevalence worldwide. It has been demonstrated that weight loss is the corner stone of NAFLD/NASH treatment. Various
options are available for weight loss in obese patients, such as life style modification, pharmacotherapy, endoscopic interventions and
bariatric/metabolic surgery. Performing bariatric/metabolic surgery only for NAFLD/NASH treatment is on debate, but it has been proven
that bariatric surgery is the most durable and effective treatment for weight loss and obesity-related comorbidities.
Keywords bariatric surgery, non-alcoholic fatty liver disease, non-alcoholic steatohepatitis, weight loss, obesity, insulin resistance, lifestyle

\section{Introduction}

Because of high fat source diet and sedentary life style which is more common recent years obesity and morbid obese population has been rapidly increased. ${ }^{1}$ According to national health and examination survey, the obesity prevalence between 20 to 74 years has been risen from 15 to $32.9 \% .^{2}$

Visceral fat accumulation in liver tissue named nonalcoholic fatty liver disease (NAFLD), known as a component of metabolic syndrome includes type 2 diabetes, hypertension and dyslipidemia represented insulin resistance (IR).

NAFLD that is considered the liver phenotype of metabolic syndrome is the most common cause of liver dysfunction and with a prevalence of $6.3-33 \%$ worldwide.

The first sign of NAFLD is accumulation of hepatic fat (steatosis) that progress to non-alcoholic steatohepatitis (NASH), liver inflammation, fibrosis, cirrhosis and hepatocellular carcinoma.

Current NAFLD and NASH treatments include weight loss obtained by life style modification and pharmaceuticals but have poor effects in patients with body mass index $>35 \mathrm{~kg} / \mathrm{m}^{2} .{ }^{5}$ Some evidences showed bariatric surgery (BS) could alter the course of disease. ${ }^{6}$ Now a days weight loss surgery considered unique method to obtain durable and permanent weight loss in morbid obesity obese could reverse metabolic syndrome consequences as well as fatty liver disease, improve liver histopathology and improve insulin resistance (IR). ${ }^{7}$ According to recent evidences we search database to approve our thesis on positive impact of BS for reversing negative metabolic syndrome effects of liver function and histopathology.

\section{Methods}

We searched PubMed, Scopus and google scholar with these at Nov 1, 2016. We found 772 papers. After deleting duplicate papers in searches (429 papers), finally 518 papers remained. 346 articles were related to our subject based on their titles and abstracts. We used only papers that their full-text was related to our title.

\section{Results}

\section{Prevalence of NAFLD and NASH in Bariatric Candidates}

NAFLD, as an emerging clinical implication, is most closely associated with obesity. ${ }^{8}$ The prevalence of obesity, as a medical and societal problem, is increasing around the world, even in developing countries. ${ }^{8,9}$ Morbid obesity (Body Mass Index (BMI) $>40 \mathrm{~kg} / \mathrm{m}^{2}$ ) is treated with the suggested weight loss interventions such as BS. ${ }^{9}$

NAFLD prevalence is much higher in obese (BMI > $30 \mathrm{~kg} / \mathrm{m}^{2}$ ) patients. ${ }^{9}$ A prospective cohort study indicated that $70 \%$ of the patients who were obese and candidate for BS, presented steatosis, a liver damage pattern of NAFLD. ${ }^{10}$ However, Results from different studies show that the prevalence of NAFLD varies between $16.7 \%$ and $96 \%$ in morbidly obese patients subjected to bariatric surgery. ${ }^{10-22}$ The wide range of NAFLD prevalence depends on the biochemical criteria and the method sensitivity used to detect NAFLD. ${ }^{8,23}$

Furthermore, NASH, as a progressive manifestation of NAFLD, occurs in $7.3 \%$ to $98 \%$ of such patients. ${ }^{10,12,13,17,18,20-22,24-30}$ The study which reported NASH in $98 \%$ of their patients, indicated that the mean super obese state (Mean BMI $52.8 \mathrm{~kg} / \mathrm{m}^{2}$ ) in their population is the reason of high NASH prevalence. ${ }^{30}$ Another study concluded that the differences in NASH prevalence can be explained by the histological criteria used to diagnose NASH. ${ }^{22}$

Ethnicity affects NAFLD prevalence as well. ${ }^{23}$ In a study on morbid obese patients underwent BS, African-American patients had lower NAFLD and NASH rate than Non-Hispanic whites and Hispanics. Non-Hispanic white and Hispanic patients had similar NAFLD prevalence. They suggested ethnic differences in fat distribution as an explanation for lower NASH rate in African-Americans. ${ }^{3}$

\section{Bariatric Surgery and Metabolic Syndrome}

Metabolic syndrome is characterized by central obesity, hypertriglyceridemia, low high density lipoprotein cholesterol (HDL-C), high blood pressure and high fasting plasma glucose. $^{32}$ 
BS is considered a safe and efficient treatment of metabolic syndrome. ${ }^{8}$ In a study of 46 morbid obese patients subjected to laparoscopic gastric bypass, metabolic syndrome and some of its components including hypertension, dysglycemia and dyslipidemia and IR were resolved after 2 years. ${ }^{33}$ Similar results were observed in a study of 827 patients who underwent laparoscopic Roux-en-Y gastric bypass (RYGB) with 4 years follow ups. ${ }^{34}$ However, improvement of metabolic syndrome is most likely associated with the amount of excess weight loss. ${ }^{35}$

\section{Insulin Resistance}

IR is considered to be the hallmark of metabolic syndrome. ${ }^{36}$ The measure of insulin sensitivity indices has shown a reduced mean insulin sensitivity in nonobese, non-diabetic young subjects with NASH in association with the different components of the metabolic syndrome.

A small study of nondiabetic severely obese patients revealed that biliopancreatic diversion (BPD) improves insulin sensitivity four days after the operation. They suggested that this sharp improvement might be explained by the intramyocellular fat depletion and enteroinsular axis interruption. ${ }^{37}$ In another study, patients who underwent gastric bypass surgery (BMI approximately $30 \mathrm{~kg} / \mathrm{m}^{2}$ ) had greater insulin sensitivity than weight-matched group $\left(\mathrm{BMI}=25\right.$ to $\left.35 \mathrm{~kg} / \mathrm{m}^{2}\right){ }^{38}$ Similarly, Klein et al. demonstrated that gastric bypass (GBP)induced weight loss markedly improves hepatic insulin sensitivity and metabolic abnormalities related to NAFLD. A decrease in intrahepatic free fatty acid (FFA) availability, caused by decreased release of FFA from intrahepatic, visceral, and subcutaneous fat is considered to be the probable explanation for marked improvement in insulin action. ${ }^{39}$

\section{Association between NAFLD and Type 2 Diabetes and the Role of Bariatric Surgery}

A study on patients who underwent jejunoileal bypass (JIB) found a decrease in fasting blood glucose and improvement in type 2 diabetes after JIB. In their study, NAFLD was observed in $86.7 \%$ of patients and NASH in $31.7 \% .^{40}$

In a retrospective study, $88.7 \%$ of patients who underwent BS and did not have diabetes and hypertension, showed liver steatosis. $7.3 \%$ of these individuals had NASH and $19.3 \%$ had liver fibrosis. Furthermore, they found liver steatosis in $96 \%$, NASH in $22 \%$ and liver fibrosis in $30.6 \%$ in the presence of diabetes and hypertension. ${ }^{20}$

In a study of 112 morbid obese patients underwent bariatric surgery, $57.7 \%$ of patients presented NASH. In these patients, type 2 diabetes was significantly $(P$ value $=0.018)$ associated with the NAFLD type 4 which is considered as $\mathrm{NASH} .{ }^{29}$

\section{Bariatric Surgery for Treatment of NAFLD/NASH in Pediatrics and Adolescents}

A recent study on obese adolescents who underwent laparoscopic sleeve gastrectomy (SG) found complete improvement of NASH in all patients and reversed hepatic fibrosis stage 2 in $90 \%{ }^{41}$

\section{The Role of Obstructive Sleep Apnea (OSA) in NAFLD and Its Improvement Following Bariatric Surgery}

In a study by cottam et al., patients with fatty liver disease and comorbid conditions underwent a two stage approach; laparoscopic SG as the first stage and laparoscopic RYGB as the second one. All cases of diabetes had improvement prior to the second stage and almost all patients with sleep apnea had resolution or improvement. Their study demonstrates that proceeding directly with a RYGB probably is not a safe option. ${ }^{42}$

A cohort study by benotti et al. showed that the severity of OSA is associated with the NAFLD severity in severe obese patients without metabolic syndrome. They concluded that the intermittent hypoxia followed by the OSA severity may be linked with the pathogenesis of NAFLD. ${ }^{43}$

\section{Indications of Bariatric Surgery in NAFLD/NASH}

NASH/NAFLD as an independent indication for bariatric/ metabolic surgery is controversial..$^{15}$

Based of new statements of IFSO (International Federation for the Surgery of Obesity and Metabolic Disorders), bariatric surgery leads to improvement and resolution of NAFLD and NASH in morbid obese patients. ${ }^{44}$

Because obesity, diabetes mellitus and hyperlipidemia (HLP) are among the risk factors for the NAFLD progression, so, weight loss induced surgery, improves these comorbidities together with NAFLD. ${ }^{45}$

Actually, the indications of surgery in NAFLD/NASH are the same as in morbid obesity and are BMI equal or more than 40 , or $35<\mathrm{BMI}<40$ with any evidence of metabolic syndrome or related comorbidities. ${ }^{5,46}$

Also bariatric surgery is indicated in overweight patients with medical-resistant severe metabolic complications. ${ }^{46}$

\section{Types of Bariatric Surgeries}

There are three different types of bariatric surgery: $:^{8,47-49}$

1. Restrictive procedures that create a state of satiety and decrease the gastric capacity and including:

a. Sleeve gastrectomy (SG): Creation of a lesser curve based narrow gastric tube, on a sizer bougie from 4-6 cm of pylorus up to gastroesophageal junction and resection of about $80 \%$ of stomach.

b. Adjustable gastric banding (AGB): Placement of an adjustable band around the upper part of stomach to create a small pouch.

c. Vertical banded gastroplasty (VBG): Creation of stapled upper part of stomach in lesser curvature that is supported by an extra luminal band.

2. Malabsorptive procedures, such as biliopancreatic diversion with or without duodenal switch (BPD-DS), that create a near $200 \mathrm{cc}$ tube of stomach like SG, but preserves the pylorus and cut the duodenum 3-4 cm after pylorus and then create a Roux-en-Y dudenojejunostomy with short common limb. These procedures alter the food and calorie intake and absorption.

3. Combined or mixed procedures such as Roux-en-Y Gastric bypass (RYGB), that create a stapled small proximal gastric pouch with well-nigh $30 \mathrm{cc}$ capacity that is anastomosed with two limbs of jejunum in RY fashion.

\section{Comparison the Effectiveness of Different Methods of Bariatric Surgery in NASH/NAFLD}

In general, bariatric surgery leads to weight loss, decreasing of transaminases and improvement of NAFLD. ${ }^{50}$

New studies show that bariatric surgery can improve the histologic features of steatosis, hepatocyte ballooning and lobular inflammation, but it effect in liver fibrosis is on debate. ${ }^{51}$ 
Bariatric surgery can also lead to significant improvement of liver function but if initial weight loss is too much and BMI loss is greater than $80 \%$, can worsen the liver function that is usually improved after one year after surgery. ${ }^{25}$

Continue to investigate the effect of two more common surgical methods throughout the world and comparing them with other relatively conventional methods described above.

1. RYGB: This method of surgery stimulates GLP-1 secretion that leads to significant improvement of some obesity-related syndromes, such as IR, inflammation and steatosis, that steatosis and also fibrosis improvement is due to decreasing of fat droplet accumulation in the liver and improvement of mitochondrial function. ${ }^{52}$

Also RYGB can correct the bile secretion derangement. ${ }^{53}$ The PYY (peptide YY) hormone secretion is increased and ghrelin hormone is decreased in this method and along with Glp-1 secretion induces the satiety state. ${ }^{54}$

There are some evidences that confirm a strong association between liver fat content reduction and IR correction in RYGB even before remarkable weight loss. ${ }^{55}$

Víctor Vargas et al. performed one liver biopsy during RYGB and another biopsy in percutaneous method, 12-22 months after surgery. They found significant histopathologic improvement in liver steatosis, ballooning degeneration, portal inflammation and fibrosis that were statistically significant. ${ }^{48}$

Joshua S Winder et al, in a study in USA, reported NAFLD improvement based on Computed Tomography (CT) scan, in $84.2 \%$ of patients after LRYGB. ${ }^{56}$

Another study that is done by Jeanne M. Clark, et al., reported improvement of all morphologic indicators of NAFLD after RYGB and $81 \%$ of patients had complete remission of liver steatosis and there were no worsening of these indicators. ${ }^{5}$

Carlos K Furuya Jr, et al., in a study performed percutaneous liver biopsy, 2 years after RYGB and reported significant improvement of steatosis (89\%), liver fibrosis (75\%) and hepatocellular ballooning (50\%) after surgery.

Everton Cazzo et al., found improvement of advanced liver fibrosis in $55 \%$ of patients, one year after LRYGB that were statistically correlated with female gender, EWL percentage, post op BMI, post op platelet and improvement of T2DM..$^{58}$

Kevin B. Barker et al., in their study, resulted in significant improvement of steatosis, lobular inflammation and periportal fibrosis and improvement of NASH in $89 \%$ of patients after RYGB. ${ }^{59}$

The study of Xiuli Liu et al., liver biopsy, showed significant improvement in macro steatosis (97\%), micro steatosis (87\%), hepatocellular ballooning (100\%) and regression of liver fibrosis, about 18 months after RYGB and notified that rapid weight loss, not only not lead to worsening of hepatocellular injuries and progression of fibrosis, but also can lead to improvement of steatosis. ${ }^{60}$

Soraya Rodrigues de Almeida, et al, in a study, notified complete improvement of NAFLD in $93.7 \%$ and lobular inflammation in $100 \%$ of patients after nearly 23.5 months after RYGB. ${ }^{61}$

2. Sleeve gastrectomy (SG): Sleeve gastrectomy is another effective method for weight loss and NAFLD improvement.
In a retrospective study, Ardeshir Algooneh et al., reported complete remission of NAFLD in $56 \%$ of patients and had a strong correlation with post op BMI, that most of NAFLD remissions were occurred in post op BMI: $25-30 .{ }^{62}$

Melania Manco et al., found 100\% improvement of NASH in adolescents, one year after SG and in their study, SG was most effective than lifestyle change and intragastric weight loss device. $^{41}$

Another study resulted in improvement of liver steatosis (66.6\%), fibrosis (68\%) and NAFLD activity score, 3 months after SG. ${ }^{63}$

\section{Comparing SG with RYGB}

A recent study, in 35 patients with $\mathrm{T} 2 \mathrm{DM}$ and $\mathrm{BMI}>35$, demonstrated that after 12 months after surgery, SG was significantly most effective on LFTs than RYGB ( $P: 0.007)$, also had better effect on NAFLD improvement, in spite of the fact that glycemic control were the same. ${ }^{64}$

Another study displayed better effect of alanine aminotransferase (ALT) improvement after SG in comparison with RYGB, but more impact for RYGB in liver fibrosis and NAFLD regression. ${ }^{6}$

\section{Comparing RYGB and LAGB}

In a study in France, who performed in 109 patients, the investigators found that RYGB resulted in more statistically significant effect in weight loss and NASH improvement in comparison with Laparoscopic Adjustable Banding (LAGB). ${ }^{46}$

Another study in 1236 patients, between 1996 to 2012, was done with one and five years follow ups, disclosed that RYGB had significantly better effects on NAFLD and liver histology that LAGB. ${ }^{66}$

\section{Other Surgical Methods}

VBG

Charalabos Stratopoulos, et al., in a study in 51 patients, that underwent VBG, with liver biopsy, 18 months after surgery demonstrated improvement of steatohepatitis un $86.2 \%$ patients, but unchanged liver fibrosis in $41.1 \%$ of cases and worsening of liver fibrosis in $11.7 \%$ of patients, however they didn't see any progression to cirrhosis. ${ }^{30}$

\section{Duodenal Switch (DS)}

Ara Keshishian et al., in a study showed that DS lead to improvement of liver steatosis and inflammation, but the patients had a transient worsening in Aspartate Aminotransferase (AST) and ALT levels, in 6 month after surgery that became normal after one year. In this study after 3 years follow up, the patient had progressive improvement of NASH. ${ }^{67}$

\section{Complications of bariatric surgery}

\section{General complications}

Different degrees of deficiencies in Iron, folate, vitamins and trace elements are seen in all bariatric surgeries. ${ }^{68}$

Gastric and anastomosis ulcers, bile reflux, dumping syndrome, adhesions, nausea and vomiting, wound infection, pulmonary emboli, abscess, inadequate weight loss and weight regain, may be present in many of bariatric procedures. ${ }^{69}$

Also in some patients that have poor response to bariatric surgery, mild increasing in liver fibrosis may occur. ${ }^{70}$ 


\section{Procedure specific complications}

1. LAGB: Band slippage, band erosion, band migration, gastric pouch dilatation and malfunction of band's port and tube are some of LAGB specific complications. ${ }^{49}$

2. SG: Bleeding, leak, GERD, antral stenosis, functional stenosis, portal thrombosis and renal failure are some of Laparoscopic Sleeve Gastrectomy (LSG) related complications. ${ }^{42,49,71}$

3. BPD/DS: GI obstruction, leak, peptic ulcer disease, protein malnutrition, fat soluble vitamin deficiencies, nutritional derangements, transient worsening of $\mathrm{NASH}$ worsening of NAFLD and liver histology due to severe weight loss and risk of bacterial stasis and overgrowth due to blind loop syndrome are specific complications of BPD/DS. ${ }^{46,48,49,67,68,72}$

4. RYGB: GI obstruction due to internal hernia, leak, malnutrition, bleeding and leak are some specific complications of RYGB. ${ }^{49}$

\section{Potential Mechanisms of Bariatric Surgery in NAFLD/NASH Treatment}

1. Improvement of IR: Weight loss, reduction of visceral lipids, increasing of GLP-1, improvement of bile acid metabolism

2. Improvement of dyslipidemia: Decreasing of LDL, HDL, TC and increasing of HDL

3. Alterations of gut hormones: Decreasing of ghrelin and increasing of GLP-1, PYY, Oxyntomodulin and bile acids

4. Decrease of inflammation: Decreasing of Interleukins 1 and $8, \mathrm{CRP}$ and TNF- $\alpha$

5. Decrease of leptin and increase of adiponectin

6. Decrease of hepatocellular apoptosis and oxidative stress of endoplasmic reticulum

7. Weight loss and BMI loss: Improvement of these major risk factors for NAFLD/NASH

8. Desirable modifications in gut microflora ${ }^{22,45,47,49,55,73-76}$

\section{Selecting the most suitable bariatric procedure in treatment of NASH/NAFLD}

Weight loss is a mainstay and gold standard method for NAFLD/NASH treatment. ${ }^{5,62}$ Bariatric surgery procedures as other weight loss programs are effective in improvement of NASH/NAFLD, but are significantly most effective than non-surgical procedures such as, medical drugs and life style

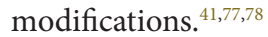

Till now, the best surgical procedure for NAFLD/NASH is not determined and meta analyses, show that all bariatric surgical procedures lead to NAFLD/NAFLD improvement in obese patients and liver steatosis in most of this patients (near 90\%). ${ }^{47,64,70,79}$

Recent studies demonstrated that bariatric surgery; result in improvement of biochemical and histologic parameters of liver, such as non-alcoholic steatosis and steatohepatitis, liver fibrosis, lobular inflammations, chronic portal inflammations and even hepatocellular carcinoma. ${ }^{47,55,62,79,80}$

Selection of bariatric surgery procedure must be done based on other conditions and indications and also need to have regular and punctual follow up after surgery. ${ }^{81}$

In confirmed NAFLD or patients that are at risk of advanced NAFLD, as possible, must avoid of malabsorptive procedures such as BPD/DS due to rapid and severe weight loss that result in FFA rapid releasing and transfer of long chain fatty acids to liver and bacterial over growth that can lead to worsening of NAFLD. ${ }^{5,48,72}$

\section{Long-term Effects of Bariatric Surgery in NAFLD/ NASH Treatment}

It seems that, bariatric surgery has durable and long standing effects in NAFLD/NASH; however, it needs to perform more related studies with longer follow ups.

Anne-Sophie Schneck et al., performed a study that had a mean 55 month follow up after RYGB, and resulted in durable improvement of liver injury, hepatocytes apoptosis, steatosis, inflammation, NAFLD activity score (NAS) and liver fibrosis in $88 \%$ of patients till that time of follow up. ${ }^{8}$

Swedish Obese Subjects (SOS) Study, that was done in retrospective fashion in a total of 3,570 obese participants, that were divided in two surgical(AGB, VBG, Bypass) and non-surgical groups, with 10 years follow ups, demonstrated improvement of transaminase levels only in surgical group that had positive correlation with weight loss. This study resulted that the short-term effects of bariatric surgery are durable and persistent in long time after surgery and have long standing protective effect against chronic liver injuries. ${ }^{50}$

\section{Non-surgical treatment (endoscopic) of NAFLD/ NASH}

Instead of bariatric surgery with different complications, there is an option to choose less invasive gadgets like endoscopic therapies with lower cost and risks ${ }^{55}$ including the endoscopic duodenal-jejunal bypass liner (DJBL) ${ }^{83}$ intragastric balloon therapy, ${ }^{55}$ and the bioenterics intragastric balloon (BIB) are present.

DJBL first motivation was to treat obesity, however, it turned out that it also leads to: ${ }^{83}$

1. Significant weight loss

2. Improvement of type 2 diabetes

3. Decrease of plasma liver parameters (normalizing of AST, ALT, andg-GT and caspasecleaved CK-18) which indicates regression of $\mathrm{NASH}$

Genco A, showed that $\mathrm{BIB}$ reduces $\mathrm{BMI}^{84}$ and improvement of metabolic profile ${ }^{85}$ even though there hasn't been any researches done on NAFLD status. it could also be used in patients with lower levels of obesity. ${ }^{49,86}$

\section{Response to bariatric surgery (biopsy, MRI, CT)}

\section{Biopsy}

Biopsy As the gold standard ${ }^{10}$ has different complications, including bleeding, pain, hypotension, hemorrhage, bile peritonitis, pneumothorax, hemothorax, transient bacteremia, hemobilia, tumor seeding and death. ${ }^{87}$ The size of the biopsy obtained can be a better representive of liver tissue. ${ }^{88}$

\section{Computer tomography}

Suitable for steatosis but not for visualizing inflammation of liver and early stages of fibrosis. ${ }^{10}$ Since weight of scanner could be a limitation, CT might not be achievable..$^{25}$

MRI

Able to detect hepatic triglyceride (hepatic fat), concentration in NAFLD and monitor liver steatosis, as non-invasive 
technique following bariatric surgery (BS), after one year a decrease in liver fat was seen..$^{89}$

\section{LiMAx test}

It can determine capacity of enzymatic liver function based on hepatic $13 \mathrm{C}$-methacetin metabolism by the cytochrome P450 $1 \mathrm{~A} 2$ system. repetitive testing will monitor disease course and response to $\mathrm{BS} .^{25}$

\section{Transient hepatic elastrography}

Fibrosis of liver has a significant relation with elasticity that is detected by this new method..$^{25}$

\section{Effectiveness of Surgical and Non-Surgical Ways of Weight Reduction in NAFLD Treatment}

Before any recommendations, since weight loss should be included as background therapy in every protocol for NAFLD treatment. $^{90}$

Bariatric surgery (BS): As average, 12 months after BS, BMI decreased to $11.9 \mathrm{~kg} / \mathrm{m}$ and NASH was cleared in $85 \%$ of patients.

Life style alteration: a healthy lifestyle results in improvement of NASH and macroinflammation ${ }^{91}$ also a reduction in oxidative stress. ${ }^{92}$ In a Cuban population a $5 \%$ reduction of weight and $25 \%$ resolution of NASH was seen. ${ }^{93}$ In a study done by St George patients were randomized in three groups moderate (6 sessions), low (3 sessions) intensity and control, a remarkable decrease of liver enzymes were seen in moderate intensity alteration $^{94}$ but the stage of fibrosis is yet to be experimented.

- Dietary limitation: By MRI measurement every 5\% decrease in BMI equals $25 \%$ in liver fat induced by hypocaloric diet. ${ }^{90}$

- Physical activity: day to day used calories by physical activity are restricted compared with what is achieved by dietary limitation. When it is integrated with diet from the starting point of behavior treatment, it might have more favorable outcomes, ${ }^{90}$ such as reduction of hepatic steatosis, liver lipids and improvement of insulin resistance. ${ }^{95}$

- Behavioral therapy.

It is burdensome for the greater part of individuals to change their life style, including their diet and physical activity. ${ }^{90}$ Weight regain is not uncommon after medical treatment. Lifestyle modifications when combined with pharmacotherapy have more favorable outcomes. ${ }^{96}$ Probiotics as regulators for energy hemostasis are associated with better liver enzyme status and histology. ${ }^{97}$

- Statins can be used in patients with dyslipidemia and NAFLD to reduce steatosis. ${ }^{98}$

- Orlistat: it decreases BMI, but has gastrointestinal side effects like, flatus and diarrhea. ${ }^{99}$

- PUFA: improves both liver stestosis and biochemical features of NAFLD. ${ }^{100}$

- Vitamin E: it is not recommended in diabetic patients or those with cirrhosis, it decreases the activity of hepatic profibrogenic actions. ${ }^{101}$

\section{What lies ahead of bariatric surgery in NAFLD patients?}

Several retrospective and prospective cohort studies has been done to clarify the optimal surgical treatment, nevertheless the gap that requires filling, is the Randomized Clinical Trial (RCT) part. Since nowadays bariatric surgery is used as a new treatment for NAFLD in obese patients, we need more longterm evidences (10 year outcomes), to find the most suitable procedure and estimation of its costs.

\section{Nutrient Deficiency and Rapid Weight Loss? A Negative Factor of BS, How to Prevent?}

Type of surgical procedure, pre operative deficiencies, continuous post operative vomiting, modified eating habits and food intolerance could have direct relation with the risk of nutritional deficiency after BS, so, patients should be monitored for rapid weight loss after BS especially with alarm symptoms such as: nausea, vomiting, and abdominal pain. ${ }^{102-104}$ Also vitamins and supplement malabsorption, can lead to nutritional deficiencies. ${ }^{105}$ Anemia due to iron, folic acid and vitamins B12, C and D (50-80\% of BS patients) $)^{106}$ deficiencies $^{107}$ and Iron deficiency which is common after gastric bypass. ${ }^{108}$ There are many concerns that rapid weight loss, may have a role in worsening of NAFLD, ${ }^{102,109}$ however Mathorin reported that in $95.7 \%$ of patients fibrosis score was not higher than $1 .{ }^{110}$ To prevent the post operative effects and improve long-term outcomes of BS, it is obligatory to: 1 . Screening of nutritional state 2. Prevention of nutrient deficiencies by prescribing appropriate supplementation, in pre and post-surgical stages. ${ }^{106}$

\section{Alternatives}

Managing patients with NAFLD contains treatment of liver disease and metabolic comorbidities, that focuses on weight loss which are achieved through hypocaloric diet or increased physical activity. ${ }^{111}$ Also daily usage of vitamin E improves histology of liver in non-diabetic adults with biopsy-proven $\mathrm{NASH}$, therefore it is not recommended for patients with NASH cirrhosis, or cryptogenic cirrhosis. ${ }^{112}$

Unfortunately, there is inadequate evidences to support metformin, thiazolidinediones, bile acids, or antioxidant supplements for NAFLD treatment.

\section{Bariatric Surgery and Long-term Effect on Liver Function Tests}

- ALT, AST, caspase-cleaved CK-18 and GGT decreased 3 months after duodenal-jejunal bypass and only permanent reduction in ALT and GGT was seen in 6 months later. $^{83}$

- Intragastric balloon and minimum weight loss of $10 \%$ decrease ALT and Gamma-Glutamyl Transferase (GGT) in a study by Ricci et al.

- Patient undergoing BPD-DS and RYGB after 3 years showed sustained reduction in BMI, ALT, and GGT. but decrease in platelet counts only occur in BPD-DS group which reflects decrease in liver fat content related inflammation and lower secondary thrombocytosis. ${ }^{113}$

- Significant reduction in ALT and AST seen in Vergas et al. study by restrictive malabsorptive procedures. ${ }^{48}$

- Preoperative AST levels and excess weight loss were associated with improvement in NASH grade score. There was no significant difference in improvement in stage between the restrictive and gastric bypass group. But there was a trend for better results in the bypass group. ${ }^{114}$

- Significant improvement in NASH was detected by the enzymatic capacity of cytochrome P450 1A2. ${ }^{25}$ 
- Short-term worsening of AST and ALT levels at first 6 months after duodenal switch operation seen in Keshishian et al. study which normalize in 6 months later. ${ }^{67}$

\section{Changes of liver histopathology after bariatric surgery}

- Overall improvement in NAFLD aspects was reported after BS also improvement in NASH and its related parameters ${ }^{116}$ but portal abnormality remains unchanged in Dixon et al. study. ${ }^{115}$

- Restrictive mildly malabsorptive (gastric bypass) procedures caused improvement in steatosis, ballooning degeneration, Mallory bodies, glycogen nuclei, Lobular inflammation, portal inflammation, and fibrosis. In a study 5 of 26 patient fibrosis persisted and $15.3 \%$ of them had $\mathrm{NASH}$ in second biopsy. ${ }^{48}$

- After 2 years of RYGB and weight loss of $60 \%$ steatosis and fibrosis disappeared with no worsening and liver biochemical markers found within normal.

- RYGB improves lobular inflammation, portal, and lobular fibrosis in Barker et al. study. There were no significant differences in serum aminotransferase levels. Two of nineteen patients still had histopathological features of NASH. One had no significant difference in portal inflammation evidence of cirrhosis and had two points improvement in steatosis and inflammation scores, the second still had evidence of mild NASH. This case had weight loss of 54 and $55 \mathrm{~kg}$, respectively, over 2 year period prior to follow-up biopsy. ${ }^{59}$

- Excess weight loss of $66 \%$ as a result of VBG leads to steatohepatitis improvement which correlated with reduction of BMI and ALT. Although overall decrease in fibrosis, $41 \%$ didn't change in fibrosis and $11.7 \%$ increase in fibrosis scores. ${ }^{30}$

- Major improvements in lobular steatosis, necroinflammatory changes, and fibrosis after laparoscopic adjustable gastric band placement in Dixon et al. occurred. In this study, no progression of histological parameters occurred. ${ }^{115}$

- LRYGB significantly improves the overall centrilobular/ perisinusoidal fibrosis scores and also induces regression of centrilobular/perisinusoidal and stage of fibrosis in NASH. However, no changes were noted in fibrosis or inflammation in the portal area after rapid weight loss after LRYGB and no worsening of hepatocellular injury or fibrosis. ${ }^{22,57,61,114}$

- De almedia et al. observed 1 complete remission, 1 fibrosis improvement and 2 cases without any changes in initial liver biopsy among 4 patients presenting fibrosis preoperatively. ${ }^{61}$

- In study of Mathurin et al. with Five years follow up almost all patients had low levels of NAFLD. Slightly increase in fibrosis and long-term improvements in Steatosis and ballooning that predicted by IR levels was seen. ${ }^{117}$

- Lobular fibrosis scores had two stages improvement in 2/19 patients and one stage in 8/19 patients. Only in one patient lobular fibrosis get worse. ${ }^{59}$

- Hepatic left lobe volume as hepatic fat indicator reduces after weight loss achieved by LAGB. ${ }^{118}$
- Duodenojejunal Bypass surgery in rats nourished by western diet normalized serum Triglyceride (TG) and attenuated accumulation of TG and steatosis in the liver of.

\section{Impact of Bariatric Surgery on Inflammation Markers}

- Serum CD163 levels increase with severity of NAFLD and reduce after weight loss even in severe liver injury indicating reversibility of macrophage activation. ${ }^{119}$

- Soluble CD14 receptor (sCD14) and human neutrophil alpha-defensins (HNDs) known as participants in hepatic necro inflammation process reduce following biliopancreatic diversion markedly correlated with improvement in NAS score. ${ }^{120}$

- Fibrogenesis regulating factors and regulating inflammation markers which observed one year after gastric bypass such as hepatic collagen-1(I), TGF- $\beta 1, \alpha-S M A$, and TIMP-1 expression, hepatic $\alpha$-SMA, hepatic expression of chemokines, MCP-1, and IL- 8 declined. At this time marked reduction in steatosis but no change in histologic assessments of inflammation and fibrosis was noted. ${ }^{39}$

- Three protein peaks observed in obese serum belongs to hemoglobin subunits increased significantly based on severity of liver lesions (steatosis and NASH) returned to normal values after bariatric surgery. None of them was correlated with metabolic parameters or LFTs. ${ }^{10}$

\section{Conclusion}

The pravalence of NAFLD is increasing along the prevalence of morbid obesity worldwide and may leads to NASH in many patients. It is demonstrated that weight loss is the main treatment for NAFLD/NASH.

There are some options to achieve weight loss and NAFLD improvement, such as life style modification, pharmacotherapy, endoscopic management and bariatric/metabolic surgery.

Although it is controversial to perform bariatric/metabolic surgery only for NAFLD/NASH, but it is proven that bariatric surgery is the most effective and durable treatment for weight loss and obesity-related comorbidities, also there are some evidences that bariatric surgery lead to decreasing of liver fat content and IR and improvement of metabolic syndrome, even before significant weight loss. Other studies suggest that improvement of metabolic syndrome after bariatric surgery has correlation with amount of weight loss. Also bariatric surgery with other mechanims, such as altration in gut hormones, changes in gut microflora, reduction of inflammatory state and decresing of hepatocellular apoptosis, potentially can improve the metabolic syndrome, NAFLD/NASH and liver fibrosis.

However, due to the lack of strong clinical trials and long follow-ups to evaluate the effect of bariatric surgery on NAFLD/NASH, choose of bariatric surgery and the best surgical procedure must be selected based on other indications and it needs to perform more clinical trials and cohort studies with long-term follow-ups. 


\section{References}

1. Sasaki A, Nitta H, Otsuka K, Umemura A, Baba S, Obuchi T, et al. Bariatric surgery and non-alcoholic fatty liver disease: current and potential future treatments. Front Endocrinol (Lausanne). 2014:5:164.

2. Rafiq N, Younossi ZM, editors. Effects of weight loss on nonalcoholic fatty liver disease. Seminars in liver disease; @Thieme Medical Publishers. 2008

3. Ricci G, Bersani G, Rossi A, Pigò F, De Fabritiis G, Alvisi V. Bariatric therapy with intragastric balloon improves liver dysfunction and insulin resistance in obese patients. Obes Surg. 2008;18:1438-1442.

4. Ebertz CE, Bonfleur ML, Bertasso IM, Mendes MC, Lubaczeuski C, Araujo AC, et al. Duodenal jejunal bypass attenuates non-alcoholic fatty liver disease in western diet-obese rats. Acta Cir Bras. 2014;29:609-614.

5. Rabl C, Campos GM. The impact of bariatric surgery on nonalcoholic steatohepatitis. Semin Liver Dis. 2012;32:80-91.

6. Helling TS, Helzberg JH, Nachnani JS, Gurram K. Predictors of nonalcoholic steatohepatitis in patients undergoing bariatric surgery:when is liver biopsy indicated? Surg Obes Relat Dis. 2008;4:612-617.

7. Furuya CK, de Oliveira CP, de Mello ES, Faintuch J, Raskovski A, Matsuda M, et al. Effects of bariatric surgery on nonalcoholic fatty liver disease: preliminary findings after 2 years. J Gastroenterol Hepatol. 2007:22:510-514.

8. Shaffer EA. Bariatric surgery:a promising solution for nonalcoholic steatohepatitis in the very obese. J Clin Gastroenterol. 2006;40 Suppl 1: S44-S50.

9. De Ridder RJ, Schoon EJ, Smulders JF, van Hout GC, Stockbrugger RW, Koek $\mathrm{GH}$. Review article: non-alcoholic fatty liver disease in morbidly obese patients and the effect of bariatric surgery. Aliment Pharmacol Therap. 2007:26 Suppl 2:195-201

10. Trak-Smayra V, Dargere D, Noun R, Albuquerque M, Yaghi C, Gannagé-Yared $\mathrm{MH}$, et al. Serum proteomic profiling of obese patients: correlation with live pathology and evolution after bariatric surgery. Gut. 2009;58:825-832.

11. Karimi-Sari H, Mousavi-Naeini SM, Ramezani-Binabaj M, Najafizadeh-Sari S, Mir-Jalili MH, Dolatimehr F. Prevalence of non-alcoholic fatty liver disease in morbidly obese patients undergoing sleeve bariatric surgery in Iran and association with other comorbid conditions. Jundishapur J Chron Dis Care. 2015;4.

12. Xanthakos SA, Jenkins TM, Kleiner DE, Boyce TW, Mourya R, Karns R, et al. High Prevalence of nonalcoholic fatty liver disease in adolescents undergoing bariatric surgery. Gastroenterology. 2015;149:623-634.

13. Corey KE, Stanley TL, Misdraji J, Scirica C, Pratt J, Hoppin A, et al. Prevalence and outcome of non-alcoholic fatty liver disease in adolescents and young adults undergoing weight loss surgery. Pediatr Obes. 2014;9:e91-e93.

14. Spaulding $L$, Trainer $T$, Janiec $D$. Prevalence of non-alcoholic steatohepatitis in morbidly obese subjects undergoing gastric bypass. Obes Surg. 2003;13:347-349.

15. Wolter S, Duprée A, Coelius C, El Gammal A, Kluwe J, Sauer N, et al. Influence of liver disease on perioperative outcome after bariatric surgery in a northern german cohort. Obes Surg. 2016:1-6.

16. Morita S, Neto Dde S, Morita FH, Morita NK, Lobo SM. Prevalence of non-alcoholic fatty liver disease and steatohepatitis risk factors in patients undergoing bariatric surgery. Obes Surg. 2015;25:2335-2343.

17. Beymer C, Kowdley KV, Larson A, Edmonson P, Dellinger EP, Flum DR. Prevalence and predictors of asymptomatic liver disease in patients undergoing gastric bypass surgery. Arch Surg (Chicago, III:1960). 2003:138:1240-1244.

18. Kroh M, Liu R, Chand B. Laparoscopic bariatric surgery:what else are we uncovering? Liver pathology and preoperative indicators of advanced liver disease in morbidly obese patients. Surg Endosc. 2007;21:1957-1960.

19. Ziolkowski A, Wylezol M, Kukla M, Zwirska-Korczala K, Berdowska A, Pardela $M$, et al. The comparison of scoring scales for liver biopsy assessment in morbidly obese patients undergoing bariatric surgery. Obes Surg. 2005;15:1309-1314.

20. Lee CJ, Clark JM, Asamoah V, Schweitzer M, Magnuson T, Lazo M. Prevalence and characteristics of individuals without diabetes and hypertension who underwent bariatric surgery:lessons learned about metabolically healthy obese. Surg Obes Relat Dis. 2015:11:142-146.

21. Losekann A, Weston AC, Carli LA, Espindola MB, Pioner SR, Coral GP. Nonalcoholic fatty liver disease in severe obese patients, subjected to bariatric surgery. Arq Gastroenterol. 2013;50:285-289.

22. Jaskiewicz K, Raczynska S, Rzepko R, Sledziński Z. Nonalcoholic fatty liver disease treated by gastroplasty. Dig Dis Sci. 2006;51:21-26.

23. Musso G, Gambino R, Cassader M. Non-alcoholic fatty liver disease from pathogenesis to management:an update. Obes Rev. 2010;11:430-445.

24. Harnois F, Msika S, Sabaté JM, Mechler C, Jouet P, Barge J, et al. Prevalence and predictive factors of non-alcoholic steatohepatitis (NASH) in morbidly obese patients undergoing bariatric surgery. Obes Surg. 2006;16:183-188.
25. Alizai PH, Wendl J, Roeth AA, Klink CD, Luedde T, Steinhoff I, et al. Functional liver recovery after bariatric surgery - a prospective cohort study with the LiMAx Test. Obes Surg. 2015;25:2047-2053.

26. Shalhub S, Parsee A, Gallagher SF, Haines KL, Willkomm C, Brantley SG, et al. The importance of routine liver biopsy in diagnosing nonalcoholic steatohepatitis in bariatric patients. Obes Surg. 2004;14:54-59.

27. Arun J, Jhala N, Lazenby AJ, Clements R, Abrams GA. Influence of liver biopsy heterogeneity and diagnosis of nonalcoholic steatohepatitis in subjects undergoing gastric bypass. Obes Surg. 2007;17:155-161.

28. Schild BZ, Santos LN, Alves MK. [Nonalcoholic fatty liver disease and its association with metabolic syndrome in the preoperative period in patients undergoing bariatric surgery]. Rev Assoc Med Bras. 2013:59:155-160.

29. Lima ML, Mourão SC, Diniz MT, Leite VH. Hepatic histopathology of patients with morbid obesity submitted to gastric bypass. Obes Surg. 2005;15: 661-669.

30. Stratopoulos C, Papakonstantinou A, Terzis I, Spiliadi C, Dimitriades G, Komesidou V, et al. Changes in liver histology accompanying massive weight loss after gastroplasty for morbid obesity. Obes Surg. 2005;15: 1154-1160.

31. Kallwitz ER, Guzman G, TenCate V, Vitello J, Layden-Almer J, Berkes J, et al. The histologic spectrum of liver disease in African-American, nonHispanic white, and Hispanic obesity surgery patients. Am J Gastroenterol. 2009;104:64-69.

32. Alberti KGM, Zimmet P, Shaw J. The metabolic syndrome-a new worldwide definition. The Lancet. 2005;366:1059

33. Ocón BJ, García B, Benito P, Gimeno S, García R, López P. Effect of gastric bypass on the metabolic syndrome and on cardiovascular risk. Nutricion Hospitalaria. 2009;25:67-71.

34. Ali MR, Fuller WD, Rasmussen J. Detailed description of early response of metabolic syndrome after laparoscopic Roux-en-Y gastric bypass. Surg Obes Relat Dis. 2009;5:346-351.

35. Batsis JA, Romero-Corral A, Collazo-Clavell ML, Sarr MG, Somers VK, LopezJimenez F, editors. Effect of bariatric surgery on the metabolic syndrome:a population-based, long-term controlled study. Mayo Clinic Proceedings; 2008: Elsevier.

36. Teixeira AR, Bellodi-Privato M, Carvalheira JB, Pilla VF, Pareja JC, D'Albuquerque LA. The incapacity of the surgeon to identify NASH in bariatric surgery makes biopsy mandatory. Obes Surg. 2009;19:1678-1684

37. Adami GF, Cordera R, Camerini G, Marinari GM, Scopinaro N. Recovery of insulin sensitivity in obese patients at short term after biliopancreatic diversion. J Surg Res. 2003;113:217-221.

38. Bikman BT, Zheng D, Pories WJ, Chapman W, Pender JR, Bowden RC, et al. Mechanism for improved insulin sensitivity after gastric bypass surgery. J Clin Endocrinol Metabol. 2008:93:4656-46563.

39. Klein S, Mittendorfer B, Eagon JC, Patterson B, Grant L, Feirt N, et al. Gastric bypass surgery improves metabolic and hepatic abnormalities associated with nonalcoholic fatty liver disease. Gastroenterology. 2006;130: 1564-1572.

40. Meinhardt NG, Souto KE, Ulbrich-Kulczynski JM, Stein AT. Hepatic outcomes after jejunoileal bypass:is there a publication bias? Obes Surg. 2006;16: $1171-1178$.

41. Manco M, Mosca A, De Peppo F, Caccamo R, Cutrera R, Giordano U, et al. The benefit of sleeve gastrectomy in obese adolescents on nonalcoholic steatohepatitis and hepatic fibrosis. J Pediat. 2016.

42. Cottam D, Qureshi FG, Mattar SG, Sharma S, Holover S, Bonanomi G, et al. Laparoscopic sleeve gastrectomy as an initial weight-loss procedure for high-risk patients with morbid obesity. Surg Endosc. 2006;20:859-863.

43. Benotti P, Wood GC, Argyropoulos G, Pack A, Keenan BT, Gao X, et al. The impact of obstructive sleep apnea on nonalcoholic fatty liver disease in patients with severe obesity. Obesity (Silver Spring, Md). 2016;24: 871-877.

44. De Luca M, Angrisani L, Himpens J, Busetto L, Scopinaro N, Weiner R, et al. Indications for surgery for obesity and weight-related diseases: position statements from the international federation for the surgery of obesity and metabolic disorders (IFSO). Obes Surg. 2016;26:1659-1696.

45. Taitano AA, Markow M, Finan JE, Wheeler DE, Gonzalvo JP, Murr MM Bariatric surgery improves histological features of nonalcoholic fatty liver disease and liver fibrosis. J Gastrointest Surg. 2015;19429-19436.

46. Lassailly G, Caiazzo R, Buob D, Pigeyre M, Verkindt H, Labreuche J, et al. Bariatric surgery reduces features of nonalcoholic steatohepatitis in morbidly obese patients. Gastroenterology. 2015:149:379-388.

47. Holterman A, Gurria J, Tanpure S, DiSomma N. Nonalcoholic fatty liver disease and bariatric surgery in adolescents. Semin Pediatr Surg. 2014;23:49-57. 
48. Vargas V, Allende H, Lecube A, Salcedo MT, Baena-Fustegueras JA, Fort $J M$, et al. Surgically induced weight loss by gastric bypass improves non alcoholic fatty liver disease in morbid obese patients. World J Hepatol. 2012:4:382-388.

49. Nobili V, Vajro P, Dezsofi A, Fischler B, Hadzic N, Jahnel J, et al. Indications and limitations of bariatric intervention in severely obese children and adolescents with and without nonalcoholic steatohepatitis: ESPGHAN hepatology committee position statement. J Pediatr Gastroenterol Nutr. 2015;60:550-561.

50. Burza MA, Romeo S, Kotronen A, Svensson PA, Sjöholm K, Torgerson JS, et al. Long-term effect of bariatric surgery on liver enzymes in the Swedish Obese Subjects (SOS) study. PLoS One. 2013;8:e60495.

51. Than NN, Newsome PN. Non-alcoholic fatty liver disease:when to intervene and with what. Clinical medicine (London, England). 2015;15:186-190.

52. Baraille F, Guilmeau S, Postic C. Gastric bypass surgery in NASH: a major modulator of hepatic mitochondrial dysfunction. Gut. 2015;64:524-526.

53. Salgado Júnior W, Donadelli CA, Dos Santos JS, Nonino CB. Influence of Roux-en-Y gastric bypass on the hepatocellular function and bile flow of obese patients assessed by scintigraphy with DISIDA. Obes Surg. 2016:26:2718-2723.

54. le Roux CW, Aylwin SJ, Batterham RL, Borg CM, Coyle F, Prasad V, et al. Gut hormone profiles following bariatric surgery favor an anorectic state, facilitate weight loss, and improve metabolic parameters. Ann Surg. 2006:243:108-114.

55. Aguilar-Olivos NE, Almeda-Valdes P, Aguilar-Salinas CA, Uribe M, MéndezSánchez $\mathrm{N}$. The role of bariatric surgery in the management of nonalcoholic fatty liver disease and metabolic syndrome. Metab Clin Exp. 2016;65: 1196-1207.

56. Winder JS, Dudeck BS, Schock S, Lyn-Sue JR, Haluck RS, Rogers AM. Radiographic improvement of hepatic steatosis after laparoscopic Rouxen-Y gastric bypass. Obes Surg. 2016.

57. Clark JM, Alkhuraishi AR, Solga SF, Alli P, Diehl AM, Magnuson TH. Roux-en-Y gastric bypass improves liver histology in patients with non-alcoholic fatty liver disease. Obes Res. 2005;13:1180-1186.

58. Cazzo E, Jimenez LS, Pareja JC, Chaim EA. Effect of Roux-en-Y gastric bypass on nonalcoholic fatty liver disease evaluated through NAFLD fibrosis score: a prospective study. Obes Surg. 2015;25:982-985.

59. Barker KB, Palekar NA, Bowers SP, Goldberg JE, Pulcini JP, Harrison SA. Nonalcoholic steatohepatitis:effect of Roux-en-Y gastric bypass surgery. Am J Gastroenterol. 2006;101:368-373.

60. Liu X, Lazenby AJ, Clements RH, Jhala N, Abrams GA. Resolution of nonalcoholic steatohepatits after gastric bypass surgery. Obes Surg. 2007;17:486-492.

61. de Almeida SR, Rocha PR, Sanches MD, Leite VH, da Silva RA, Diniz MT, et al. Roux-en-Y gastric bypass improves the nonalcoholic steatohepatitis (NASH) of morbid obesity. Obes Surg. 2006;16:270-278.

62. Algooneh A, Almazeedi S, Al-Sabah S, Ahmed M, Othman F. Non-alcoholic fatty liver disease resolution following sleeve gastrectomy. Surg Endosc. 2016:30:1983-1987.

63. Aldoheyan T, Hassanain M, Al-Mulhim A, Al-Sabhan A, Al-Amro S, Bamehriz $F$, et al. The effects of bariatric surgeries on nonalcoholic fatty liver disease. Surg Endoscopy. 2016.

64. Billeter AT, Senft J, Gotthardt D, Knefeli P, Nickel F, Schulte T, et al. Combined non-alcoholic fatty liver disease and type 2 diabetes mellitus: sleeve gastrectomy or gastric bypass?-a controlled matched pair study of 34 patients. Obes Surg. 2016;26:1867-1874.

65. Froylich D, Corcelles R, Daigle C, Boules M, Brethauer S, Schauer P. Effect of Roux-en-Y gastric bypass and sleeve gastrectomy on nonalcoholic fatty liver disease:a comparative study. Surg Obes Relat Dis. 2016;12:127-131.

66. Caiazzo R, Lassailly G, Leteurtre E, Baud G, Verkindt H, Raverdy V, et al. Roux-en- $Y$ gastric bypass versus adjustable gastric banding to reduce nonalcoholic fatty liver disease:a 5-year controlled longitudinal study. Annals of surgery. 2014;260:893-898.

67. Keshishian A, Zahriya K, Willes EB. Duodenal switch has no detrimental effects on hepatic function and improves hepatic steatohepatitis after 6 months. Obes Surg. 2005;15:1418-1423.

68. Miras AD, le Roux CW. Metabolic surgery:shifting the focus from glycaemia and weight to end-organ health. Lancet Diabetes Endocrinol. 2014;2:141-151.

69. Polymeris $A$. The pluses and minuses of bariatric surgery for morbid obesity:An endocrinological perspective. Hormones (Athens). 2012;11: 233-240.

70. Lassailly G, Caïazzo R, Pattou F, Mathurin P. Bariatric surgery for curing NASH in the morbidly obese? J Hepatol. 2013;58:1249-1251.
71. Boza C, Salinas J, Salgado N, Pérez G, Raddatz A, Funke R, et al. Laparoscopic sleeve gastrectomy as a stand-alone procedure for morbid obesity: report of 1,000 cases and 3-year follow-up. Obes Surg. 2012;22:866-871.

72. Vander Naalt SJ, Gurria JP, Holterman AL. Surgical treatment of nonalcoholic fatty liver disease in severely obese patients. Hepat Med. 2014;6:103-112.

73. Sasaki A, Nitta H, Otsuka K, Umemura A, Baba S, Obuchi T, et al. Bariatric surgery and non-alcoholic fatty liver disease:Current and potential future treatments. Front Endocrinol. 2014:5.

74. Hafeez S, Ahmed MH. Bariatric surgery as potential treatment for nonalcoholic fatty liver disease:a future treatment by choice or by chance? J Obes. 2013:2013:839275.

75. Mosinski JD, Pagadala MR, Mulya A, Huang H, Dan O, Shimizu H, et al. Gastric bypass surgery is protective from high-fat diet-induced nonalcoholic fatty liver disease and hepatic endoplasmic reticulum stress. Acta physiol (Oxford, England). 2016:217:141-151.

76. Dietrich P, Hellerbrand C. Non-alcoholic fatty liver disease, obesity and the metabolic syndrome. Best Practice and Research: Clin Gastroenterol. 2014;28:637-653.

77. Myronovych A, Kirby M, Ryan KK, Zhang W, Jha P, Setchell KD, et al. Vertical sleeve gastrectomy reduces hepatic steatosis while increasing serum bile acids in a weight-loss-independent manner. Obesity (Silver Spring, Md). 2014:22:390-400

78. Milić S, Lulić D, Štimac D. Non-alcoholic fatty liver disease and obesity:biochemical, metabolic and clinical presentations. World J Gastroenterol. 2014;20:9330-9337.

79. Watanabe S, Hashimoto E, Ikejima K, Uto H, Ono M, Sumida Y, et al; Japanese Society of Gastroenterology; Japan Society of Hepatology. Evidence-based clinical practice guidelines for nonalcoholic fatty liver disease/nonalcoholic steatohepatitis. J Gastroenterol. 2015:50:364-377.

80. Neuman MG, Nanau RM, Cohen LB. Nonmedicinal interventions in nonalcoholic fatty liver disease. Can J Gastroenterol Hepatol. 2015;29: 241-252.

81. Schneier AT, Citti CC, Dieterich DT. Management and diagnosis of fatty liver disease. Expert Rev Gastroenterol Hepatol. 2015;9:671-683.

82. Schneck AS, Anty R, Patouraux S, Bonnafous S, Rousseau D, Lebeaupin C, et al. Roux-En Y gastric bypass results in long-term remission of hepatocyte apoptosis and hepatic histological features of non-alcoholic steatohepatitis. Front Physiol. 2016;7:344.

83. de Jonge C, Rensen SS, Koek GH, Joosten MF, Buurman WA, Bouvy ND, et al. Endoscopic duodenal-jejunal bypass liner rapidly improves plasma parameters of nonalcoholic fatty liver disease. Clin Gastroenterol Hepatol. 2013;11:1517-1520.

84. Genco A, Bruni T, Doldi SB, Forestieri P, Marino M, Busetto L, et al. Bioenterics Intragastric balloon: the Italian experience with 2,515 patients. Obes Surg. 2005:15:1161-1164

85. Totté E, Hendrickx L, Pauwels M, Van Hee R. Weight reduction by means of intragastric device: experience with the bioenterics intragastric balloon. Obes Surg. 2001;11:519-523

86. Doldi S, Micheletto G, Perrini M, Rapetti R. Intragastric balloon: another option for treatment of obesity and morbid obesity. Hepato-gastroenterology. 2003:51:294-297.

87. Nassif AT, Nagano TA, Okayama S, Nassif LS, Branco Filho A, Sampaio Neto J. Performance of the bard scoring system in bariatric surgery patients with nonalcoholic fatty liver disease. Obes Surg. 2016.

88. Larson SP, Bowers SP, Palekar NA, Ward JA, Pulcini JP, Harrison SA. Histopathologic variability between the right and left lobes of the liver in morbidly obese patients undergoing Roux-en-Y bypass. Clin Gastroenterol Hepatol. 2007:5:1329-1332.

89. Jiménez-Agüero R, Emparanza Jl, Beguiristain A, Bujanda L, Alustiza JM, García E, et al. Novel equation to determine the hepatic triglyceride concentration in humans by MRl:diagnosis and monitoring of NAFLD in obese patients before and after bariatric surgery. BMC Med. 2014;12:137.

90. Marchesini G, Petta S, Dalle Grave R. Diet, weight loss, and liver health in nonalcoholic fatty liver disease:Pathophysiology, evidence, and practice. Hepatology (Baltimore, Md). 2016:63:2032-2043.

91. Promrat K, Kleiner DE, Niemeier HM, Jackvony E, Kearns M, Wands JR, et al. Randomized controlled trial testing the effects of weight loss on nonalcoholic steatohepatitis. Hepatology (Baltimore, Md). 2010;51: 121-129.

92. Angelico F, Loffredo L, Pignatelli P, Augelletti T, Carnevale R, Pacella A, et al. Weight loss is associated with improved endothelial dysfunction via NOX generated oxidative stress down-regulation in patients with the metabolic syndrome. Intern Emerg Med. 2012;7:219-227. 
93. Vilar-Gomez E, Martinez-Perez Y, Calzadilla-Bertot L, Torres-Gonzalez A, Gra-Oramas B, Gonzalez-Fabian L, et al. Weight loss through lifestyle modification significantly reduces features of nonalcoholic steatohepatitis. Gastroenterology. 2015;149:367-378. e5.

94. St George A, Bauman A, Johnston A, Farrell G, Chey T, George J. Effect of a lifestyle intervention in patients with abnormal liver enzymes and metabolic risk factors. J Gastroenterol Hepatol. 2009;24:399-407.

95. Hallsworth K, Fattakhova G, Hollingsworth KG, Thoma C, Moore S, Taylor $\mathrm{R}$, et al. Resistance exercise reduces liver fat and its mediators in nonalcoholic fatty liver disease independent of weight loss. Gut. 2011:242073.

96. Marchesini G, Mazzella N, Forlani G. Weight Loss for a Healthy Liver. Gastroenterology. 2015;149:274-278.

97. Iacono A, Raso GM, Canani RB, Calignano A, Meli R. Probiotics as an emerging therapeutic strategy to treat NAFLD: focus on molecular and biochemical mechanisms. J Nutr Biochem. 2011;22:699-711.

98. Ekstedt M, Franzén LE, Mathiesen UL, Holmqvist M, Bodemar G, Kechagias S. Statins in non-alcoholic fatty liver disease and chronically elevated liver enzymes:a histopathological follow-up study. J Hepatol. 2007:47:135-141.

99. Ioannides-Demos LL, Piccenna L, McNeil JJ. Pharmacotherapies for obesity:past, current, and future therapies. J Obesity. 2010;2011.

100. Capanni M, Calella F, Biagini MR, Genise S, Raimondi L, Bedogni G, et al. Prolonged $n-3$ polyunsaturated fatty acid supplementation ameliorates hepatic steatosis in patients with non-alcoholic fatty liver disease:a pilot study. Aliment Pharmacol Ther. 2006;23:1143-1151.

101. Attar BM, Van Thiel DH. Current concepts and management approaches in nonalcoholic fatty liver disease. Scientific World Journal. 2013;2013:481893.

102. Younossi ZM, Reyes MJ, Mishra A, Mehta R, Henry L. Systematic review with meta-analysis:non-alcoholic steatohepatitis - a case for personalised treatment based on pathogenic targets. Aliment Pharmacol Ther. 2014;39:3-14.

103. Dagan SS, Zelber-Sagi S, Webb M, Keidar A, Raziel A, Sakran N, et al. Nutritional status prior to laparoscopic sleeve gastrectomy surgery. Obes Surg. 2016;26:2119-2126.

104. De Palma GD, Forestieri P. Role of endoscopy in the bariatric surgery of patients. World J Gastroenterol. 2014;20:7777-7784

105. Padwal R, Brocks D, Sharma AM. A systematic review of drug absorption following bariatric surgery and its theoretical implications. Obes Rev. 2010;11:41-50

106. Luger M, Kruschitz R, Marculescu R, Haslacher H, Hoppichler F, Kallay E, et al. The link between obesity and vitamin $D$ in bariatric patients with omega-loop gastric bypass surgery - a vitamin D supplementation trial to compare the efficacy of postoperative cholecalciferol loading (LOAD): study protocol for a randomized controlled trial. Trials. 2015;16.

107. Shah M, Simha V, Garg A. Long-term impact of bariatric surgery on body weight, comorbidities, and nutritional status. J Clin Endocrinol Metabol. 2006;91:4223-4231.
108. Fleischer J, Stein E, Bessler M, Badia MD, Restuccia N, Olivero-Rivera $\mathrm{L}$, et al. The decline in hip bone density after gastric bypass surgery is associated with extent of weight loss. J Clin Endocrinol Metabol. 2008:93:3735-3740

109. Verna EC, Berk PD. Role of fatty acids in the pathogenesis of obesity and fatty liver:impact of bariatric surgery. Semin Liver Dis. 2008;28:407-426.

110. Mathurin P, Hollebecque A, Arnalsteen L, Buob D, Leteurtre E, Caiazzo $\mathrm{R}$, et al. Prospective study of the long-term effects of bariatric surgery on liver injury in patients without advanced disease. Gastroenterology. 2009;137:532-540.

111. Haentjens P, Massaad D, Reynaert H, Peeters E, Van Meerhaeghe A, Vinken $S$, et al. Identifying non-alcoholic fatty liver disease among asymptomatic overweight and obese individuals by clinical and biochemical characteristics. Acta Clin Belg 2009;64:483-493.

112. Prashanth M, Ganesh HK, Vima MV, John M, Bandgar T, Joshi SR, et al. Prevalence of nonalcoholic fatty liver disease in patients with type 2 diabetes mellitus. J Assoc Physicians India. 2009;57:205-210.

113. Johansson HE, Haenni A, Zethelius B. Platelet counts and liver enzymes after bariatric surgery. J Obes. 2013;2013:567984

114. Mattar SG, Velcu LM, Rabinovitz M, Demetris AJ, Krasinskas AM, BarinasMitchell $E$, et al. Surgically-induced weight loss significantly improves nonalcoholic fatty liver disease and the metabolic syndrome. Ann Surg. 2005;242:610-617.

115. Dixon JB, Bhathal PS, Hughes NR, O'Brien PE. Nonalcoholic fatty liver disease: improvement in liver histological analysis with weight loss. Hepatology (Baltimore, Md). 2004;39:1647-154.

116. Verbeek J, Lannoo M, Pirinen E, Ryu D, Spincemaille P, Vander Elst I, et al. Roux-en-Y gastric bypass attenuates hepatic mitochondrial dysfunction in mice with non-alcoholic steatohepatitis. Gut. 2015;64:673-683.

117. Mathurin P, Hollebecque A, Arnalsteen L, Buob D, Leteurtre E, Caiazzo R, et al. Prospective study of the long-term effects of bariatric surgery on liver injury in patients without advanced disease. Gastroenterology. 2009; 137:532-540.

118. Giannetti M, Piaggi P, Ceccarini G, Mazzeo S, Querci G, Fierabracci $P$, et al. Hepatic left lobe volume is a sensitive index of metabolic improvement in obese women after gastric banding. Int J Obes (Lond). 2012;36:336-341

119. Kazankov K, Tordjman J, Møller HJ, Vilstrup H, Poitou C, Bedossa P, et al. Macrophage activation marker soluble CD163 and non-alcoholic fatty liver disease in morbidly obese patients undergoing bariatric surgery. J Gastroenterol Hepatol. 2015;30:1293-1300.

120. Manco M, Fernandez-Real JM, Vecchio FM, Vellone V, Moreno JM, Tondolo $\checkmark$, et al. The decrease of serum levels of human neutrophil alpha-defensins parallels with the surgery-induced amelioration of NASH in obesity. Obes Surg. 2010;20:1682-1689.

This work is licensed under a Creative Commons Attribution-NonCommercial 3.0 Unported License which allows users to read, copy, distribute and make derivative works for non-commercial purposes from the material, as long as the author of the original work is cited properly. 